\title{
Analysis of the 17-segment left ventricle model using generalized estimating equations
}

\author{
Samantha R. Seals, PhD, ${ }^{\mathrm{a}}$ and Inmaculada B. Aban, PhD ${ }^{\mathrm{b}}$ \\ ${ }^{a}$ Center of Biostatistics and Bioinformatics, University of Mississippi Medical Center, Jackson, MS \\ ${ }^{b}$ Department of Biostatistics, University of Alabama at Birmingham, Birmingham, AL
}

Received May 20, 2015; accepted May 20, 2015

doi:10.1007/s12350-015-0186-4

\section{See related article, pp. 1102-1109}

Consider the problem of comparing imaging techniques where the study design is such that a participant is subjected to both techniques. If the outcome of interest is such that each technique results in only one observation per participant, most of the commonly used standard methods of statistical data analyses may be applicable. However, an added complication to the analysis occurs when the outcome of interest from the imaging techniques that are being compared results in multiple observations per subject. In particular, researchers following the 17-segment left ventricular model (recommended by the American Heart Association $^{1}$ ) who are interested in the outcomes at the segment level have to deal with 17 observations per subject in the analyses.

The paper by Oldan et al, ${ }^{2}$ published in this issue, investigates this type of scenario. The goal was to compare the results of two imaging techniques with respect to segmental uptake measurements: positron emission tomography-computed tomography (PET-CT) and positron emission tomography-magnetic resonance (PET-MR). Therefore, each participant in their study had a total of 34 correlated observations. Statistical analyses should consider both between-segment and between-techniques correlation assuming that the participants are not associated with each other.

Reprint requests: Samantha R. Seals, PhD, Center of Biostatistics and Bioinformatics, University of Mississippi Medical Center, 2500

North State Street, Jackson, MS 39216; sseals@umc.edu

J Nucl Cardiol 2016;23:1110-1.

$1071-3581 / \$ 34.00$

Copyright (c) 2015 American Society of Nuclear Cardiology.
With only two techniques to compare, taking the difference, e.g., PET-MR minus PET-CT, at each segment is one way to simplify the analyses. This method reduces the number of observations per participant from 34 to 17 and leaves only the between-segment correlation to consider. Oldan et al used this method and modeled the difference in the two techniques to examine factors (such as gender, BMI, segment) that may be related to this difference.

Standard regression models will not be applicable in this case as these methods ignore the between-segment correlation, and hence may result in inflated type I errors (i.e., erroneously concluding significant results). ${ }^{3}$ The authors chose to use a generalized linear regression model utilizing generalized estimating equations (GEE). ${ }^{4,5}$ This model handles multiple observations per subject by specifying a working covariance structure that models the correlation between segments. It is worth noting that the generalized linear model utilizing GEE may also be used if the outcome being modeled is continuous, binary, or count data.

Some common covariance structures include compound symmetric, independent, and unstructured. Compound symmetric, also known as the exchangeable structure, assumes that the variance is the same for all segments and correlation between any pair of segments is the same and has two covariance parameters to estimate. This is the covariance structure used by Olden et al. Independent, also known as variance components, assumes that all observations are independent (i.e., segments are not correlated), thus, have covariance of 0 . The independent structure is a structure that does not assume common variance and therefore estimates 17 values associated with the variances of the 17 segments. Unstructured is the most flexible and does not assume a specific functional relationship, allowing the data to speak for itself. However, this can result in a large number of covariance parameters to estimate; in the case of the 17-segment LV model, there are 136 covariance 
parameters to estimate. This large number of parameters for estimation can cause estimation problems with small sample sizes.

Another aspect of comparing two techniques is to answer the question: Are the two techniques "equivalent' with respect to an outcome of interest? Statistical methods for testing equivalence should be used, such as those proposed by Bland and Altman, ${ }^{6,7}$ drawing a scatterplot of observations from both techniques and comparing them against the $x=y$ line (a line passing through the origin with unit slope), and computing measures of correlation such as intra-class correlation ${ }^{8}$ or the concordance correlation coefficient as proposed by Lin. ${ }^{9}$ The more traditional Pearson correlation may be used as a measure of equivalence only if the scatterplot shows a close proximity to the $x=y$ line. If one technique consistently gives a higher value than the other by an almost constant amount, the Pearson correlation will be high but clearly the two techniques are not equivalent.

In Oldan et al, Figures 3, 5a, and 6a are scatterplots comparing the values from the two techniques relative to the $x=y$ line with corresponding correlation values. It is unclear what type of correlation is reported but, presumably, it is the Pearson correlation which is the standard correlation coefficient paired with the scatterplot. These graphs and correlation are good descriptive tools to see how the two techniques compare, however, when making inferences (statistical test and confidence interval estimation) about the correlation between the readings from the two techniques; it is important to use methods that account between-segment correlation such as the GEE, as previously discussed.

As a final note, if the values from the imaging techniques can be approximated by a multivariate normal distribution, one may use a generalized linear mixed model (GLMM) instead of GEE to analyze the data. GLMM will be more powerful than GEE if the assumption of multivariate normal holds. Furthermore, statistical packages such as SAS offer more working covariance structures, including spatial functions, where one can fit a distance-dependent model, which accounts for the location of the LV segments in space. In this case, the spatial working covariance structures account for the fact that segments closer to each other are more highly correlated than those farther apart. Bowman and Waller, ${ }^{10}$ George and Aban, ${ }^{11}$ and Seals et $\mathrm{al}^{3}$ show how one can apply these spatial functions to model correlation among the segments. Statistical packages such as SAS and Stata have model indices such as the Akaike
Information Criterion (AIC) $)^{12,13}$ and the Bayesian Information Criterion (BIC), ${ }^{14}$ in the case of GLMM, or the Quasi-likelihood under the Independence model Criterion (QIC or QICu), ${ }^{15,16}$ in the case of GEE. Note that because GEE does not rely on likelihood methods, AIC and BIC are not applicable. These indices may be used to choose from candidate models to find the model that provides the best fit. We note that the AIC, BIC, and QIC may be used to choose both the best working covariance structure as well as variable selection, whereas the $\mathrm{QICu}$ is only valid for variable selection.

\section{References}

1. Cerqueira MD, Weissman NJ, Dilsizian V, Jacobs AK, Kaul S, Laskey WK, et al. Standardized myocardial segmentation and nomenclature for tomographic imaging of the heart: A statement for healthcare professionals from the Cardiac Imaging Committee of the Council on Clinical Cardiology of the American Heart Association. Circulation 2002;105:539-42.

2. Oldan J, Shah S, Brunken R, DiFilippo F, Obuchowski N, Bolen M. Do myocardial PET-MR and PET-CT FDG images provide comparable information? J Nucl Cardiol. doi:10.1007/s12350-0150159-7.

3. Seals SR, Katholi CR, Zhang J, Aban IB. Evaluating the use of spatial covariance structures in the analysis of cardiovascular imaging data. Commun Stat - Simul Comput (in press).

4. Liang KY, Zeger SL. Longitudinal data analysis using generalized linear models. Biometrika 1986;73:13-22.

5. Zeger SL, Liang KY. Longitudinal data analysis for discrete and continuous outcomes. Biometrics 1986;42:121-30.

6. Bland JM, Altman DG. Statistical methods for assessing agreement between two methods of clinical measurement. Lancet 1986;1:307-10

7. Bland JM, Altman DG. Measuring agreement in method comparison studies. Stat Methods Med Res 1999;8:135-60.

8. Fisher RA. Statistical methods for research workers. London: Oliver and Boyd; 1925.

9. Lin LIK. A concordance correlation coefficient to evaluate reproducibility. Biometrics 1989;45:255-68.

10. Bowman FD, Waller LA. Modelling of cardiac imaging data with spatial correlation. Stat Med 2004;23:965-85.

11. George B, Aban I. Selecting a separable parametric spatiotemporal covariance structure for longitudinal imaging data. Stat Med 2015;34:145-61.

12. Akaike $H$. Information theory and an extension of the maximum likelihood principle. Second International Symposium on Information Theory 1973;267-81.

13. Akaike H. A new look at the statistical model identification. IEEE Trans Autom Control 1974;19:716-23.

14. Schwarz GE. Estimating the dimension of a model. Ann Stat 1978;6:461-4

15. Pan W. Akaike's information criterion in generalized estimating equations. Biometrics 2001;57:120-5.

16. Pan W. Model selection in estimating equations. Biometrics 2001;57:529-34. 